\title{
APPLICATION OF FFT AND ARMA SPECTRAL ANALYSIS TO ARTERIAL DOPPLER SIGNALS
}

\author{
Elif Derya Übeyli, Hakan Işık and İnan Güler \\ Gazi University, Faculty of Technical Education, Department of Electronics and \\ Computer Education, 06500 Teknikokullar, Ankara, Turkey \\ iguler@tef.gazi.edu.tr
}

\begin{abstract}
In this study, Doppler signals recorded from arteries of 105 subjects were processed by PC-computer using fast Fourier transform, and least squares modified Yule-Walker autoregressive moving average methods. By using these spectrum analysis techniques, the variations in the shape of the Doppler spectrums as a function of time were presented in the form of sonograms in order to obtain medical information. These sonograms were then used to compare the applied methods in terms of their frequency resolution and the effects in determination of stenosis and occlusion in arteries. Reliable information on hemodynamic alterations in arteries can be obtained by evaluation of these sonograms.
\end{abstract}

Keywords- Doppler signal, Spectral analysis, Stenosis, Occlusion

\section{INTRODUCTION}

Doppler ultrasound has been a widely used technique in clinical applications since the 1980s. Doppler systems are based on the principle that ultrasound, emitted by an ultrasonic transducer, is returned partially towards the transducer by the moving red blood cells, thereby inducing a shift in frequency proportional to the emitted frequency and the velocity along the ultrasound beam. Since the scatterers within the ultrasound beam usually do not move at the same speed, a spectrum of Doppler frequencies will be observed. By using spectrum analysis techniques, the variations in the shape of the Doppler spectrums as a function of time are presented in the form of sonograms in order to obtain medical information. Doppler ultrasound has proven to be a valuable technique for investigation of artery conditions. Doppler ultrasonography is a reliable technique, which demonstrates the flow characteristics and resistance of arteries in stenosis and occlusion conditions. The results of the studies in the literature have shown that Doppler ultrasound evaluation can give reliable information on both systolic and diastolic blood velocities of arteries and have supported that Doppler ultrasound is useful in screening certain hemodynamic alterations in arteries [1-4].

A number of spectral estimation techniques have recently been developed and have been compared to the more standard fast Fourier transform (FFT) method, or Welch method, for Doppler ultrasonic signal processing [2-4]. The classical (nonparametric) power spectrum estimation methods, originally introduced by Schuster (1898). The model-based (parametric) methods were originated by Yule (1927). These methods were subsequently developed and applied by Walker (1931), Bartlett (1948), Parzen (1957), Blackman and Tukey (1958), and other researchers [5]. One of the parametric methods is autoregressive moving average (ARMA) method. ARMA spectra can be computed via several algorithms such as least squares modified Yule-Walker method. ARMA method has been proposed for spectrum estimation in several papers, 
including the papers Graupe et al. (1975), Cadzow (1981, 1982), Kay (1980), and Friedlander (1982) [5,6].

In this study, Doppler signals obtained from 105 subjects, 38 of them have suffered from artery stenosis and 25 of them have suffered from artery occlusion, are examined by taking into consideration of their sonograms. FFT, and least squares modified Yule-Walker ARMA methods are used for spectrum analysis of Doppler signals obtained from arteries. Using these spectrum analysis techniques, the timedependent spectral distributions are visualized and detailed documentations of the Doppler signals are obtained. These methods are compared in terms of their frequency resolution and the effects in determination of stenosis and occlusion in arteries.

\section{MATERIALS AND METHOD}

\subsection{Measurements Of Doppler Signals From Arteries}

Doppler ultrasound system is used as a noninvasive method to observe the hemodynamics of arteries. Arterial examinations are performed with a Doppler unit using a $7.5 \mathrm{MHz}$ ultrasonic transducer. The block diagram of the measurement system is shown in Figure 1. The system consists of five blocks. These are $7.5 \mathrm{MHz}$ ultrasonic transducer, analog Doppler unit (Toshiba 140A Color Doppler Ultrasonography), recorder (Sony), analog/digital interface board (Sound Blaster Pro-16 bit), a personal computer with a printer [2-4].

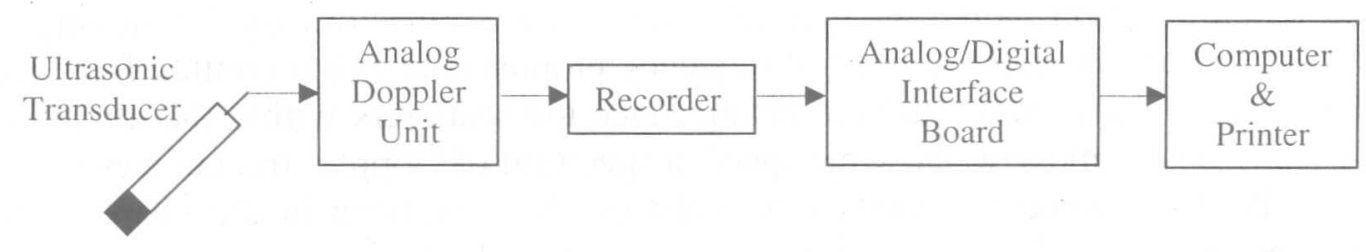

Figure 1. Block diagram of measurement system

The ultrasonic transducer is applied on a horizontal plane to the body surface using water-soluble gel as a coupling gel. Care is taken not to apply pressure to the body surface in order to avoid artifacts. The probe is most often placed at an angle of 60 degrees towards the body surface. The instrument displays Doppler frequencies as centimeters per second on the $\mathrm{y}$-axis and plotted time in seconds on the $\mathrm{x}$-axis called as sonograms. In sonograms, time is plotted along the horizontal axis, frequency along the vertical axis and the power at a particular frequency and time as the intensity of the corresponding pixel [1-4]. Arterial signals used in this study belong to healthy and unhealthy (subjects suffered from artery stenosis and occlusion) subjects. MATLAB software package (version 6.0) is used to form sonograms of Doppler signals. FFT and least squares modified Yule-Walker ARMA methods are used to obtain sonograms which represent the changes in Doppler frequency with respect to time. 


\subsection{Fast Fourier Transform Method For Spectral Analysis}

FFT methods such as Welch method are defined as nonparametric methods. Welch spectral estimator is one of the FFT methods and relies on the definition of periodogram method. If available information on the signal consists of the samples $\{x(n)\}_{n=1}^{N}$, the periodogram spectral estimator is defined as

$\hat{P}_{P E R}(f)=\frac{1}{N}\left|\sum_{n=1}^{N} x(n) \exp (-j 2 \pi f n)\right|^{2}$

Signals are divided into overlapping intervals, windowed by using Hanning window function, periodograms are calculated and then average of periodograms is found $[5,6]$. $\left\{x_{l}(n)\right\}, l=1, \ldots, S$ are signal intervals and each interval's length equals to $M$. In this method, generally overlapping ratio is taken as $50 \%(K \cong M / 2)$. Welch spectral estimator is defined as

$\hat{P}_{l}(f)=\frac{1}{M} \frac{1}{P}\left|\sum_{n=1}^{M} v(n) x_{l}(n) \exp (-j 2 \pi f n)\right|^{2} \quad$ and $\quad \hat{P}_{W}(f)=\frac{1}{S} \sum_{l=1}^{S} \hat{P}_{l}(f)$

where $v(n)$ is data window, $P$ is total average of $v(n)$ and given as $P=\frac{1}{M} \sum_{n=1}^{M}|v(n)|^{2}$.

Then, evaluation of $\hat{P}_{W}(f)$ at the frequency samples basically reduces to the computation of the following discrete Fourier transform (DFT):

$X(k)=\sum_{n=1}^{N} x(n) \exp \left(-j \frac{2 \pi}{N}\right)^{n k}, \quad k=0, \ldots, N-1$

where $X(k)$ is expressed as the discrete Fourier coefficient, $N$ is the length of available data and $x(n)$ is the input signal on the time domain. The procedure that computes equation (3) is called as FFT algorithm. The estimated Welch power spectrum density (PSD) can be computed by use of the DFT, which in turn is efficiently computed by the FFT algorithm [5,6]. FFT method is a poor spectral estimator because its variance is high and frequency resolution of FFT method is limited by the available data record duration, independent of the characteristics of the data. In addition, a smeared spectral estimate is a consequence of the windowing. Owing to these limitations of FFT method, parametric (model-based) spectral estimation methods, like ARMA method, are extremely valuable for data analysis $[5,6]$.

\subsection{ARMA Method For Spectral Analysis}

The parametric (model-based) methods are based on modeling the data sequence $x(n)$ as the output of a linear system characterized by a rational system. In the parametric methods, the spectrum estimation procedure consists of two steps. Given the data sequence $x(n), 0 \leq n \leq N-1$, the parameters of the method are estimated. Then from these estimates, the PSD estimate is computed. The spectral factorization problem associated with a rational PSD has multiple solutions, with the stable and minimum phase ARMA model being one of them. In ARMA method, data can be modeled as 
output of a causal, pole-zero, discrete filter whose input is white noise. ARMA method of order $p, q$ is expressed as the following equation:

$x(n)=-\sum_{k=1}^{p} a(k) x(n-k)+\sum_{k=0}^{q} b(k) w(n-k)$

where $a(k)$ are AR coefficients, $b(k)$ are MA coefficients and $w(n)$ is white noise of variance equal to $\sigma^{2}$. ARMA $(p, q)$ model can be characterized by ARMA parameters $\left\{a[1], a[2], \ldots, a[p], b[1], b[2], \ldots, b[q], \sigma^{2}\right\}$. The PSD is

$P_{A R M A}(f)=\sigma^{2}\left|\frac{B(f)}{A(f)}\right|^{2}$

where $A(f)=1+a_{1} e^{-j 2 \pi f}+\ldots+a_{p} e^{-j 2 \pi f p}$ and $B(f)=1+b_{1} e^{-j 2 \pi f}+\ldots+b_{q} e^{-j 2 \pi f q}$.

\subsubsection{Least Squares Modified Yule-Walker Method For ARMA Parameter Estimation}

A reliable method is to construct a set of linear equations and to use the method of least squares on the set of equations. Suppose that the autocorrelation sequence can be accurately estimated up to $\operatorname{lag} M$, where $M>p+q$. Then the following set of linear equations can be written:

$\left[\begin{array}{cccc}r(q) & r(q-1) & \cdots & r(q-p+1) \\ r(q+1) & r(q) & \cdots & r(q-p+2) \\ \vdots & \vdots & & \\ r(M-1) & r(M-2) & & r(M-p)\end{array}\right]\left[\begin{array}{c}a_{1} \\ a_{2} \\ \vdots \\ a_{p}\end{array}\right]=-\left[\begin{array}{c}r(q+1) \\ r(q+2) \\ \vdots \\ r(M)\end{array}\right]$

or equivalently,

$R a=-r$

Since dimension of $R$ is $(M-q) x p$ and $M-q>p$ the least squares criterion can be used to solve for the parameter vector $a$. The result of this minimization is

$\hat{a}=-\left(R^{*} R\right)^{-1}\left(R^{*} r\right)$

To estimate an MA spectrum is based on the reparameterization of the PSD in terms of the autocorrelation sequence. For an MA order of $q$,

$r(k)=0$ for $|k|>q$

Owing to this simple observation, the definition of the PSD as a function of $\{r(k)\}$ turns into a finite-dimensional spectral model:

$P(f)=\sum_{k=-q}^{q} r(k) \exp (-j 2 \pi f k)$

Hence a simple estimator of MA PSD is obtained by inserting estimates of $\{r(k)\}_{k=0}^{q}$ in equation (10). If the standard autocorrelation function estimates are used, then PSD estimate is obtained as 
$\hat{P}_{M A}(f)=\sum_{k=-q}^{q} \hat{r}(k) \exp (-j 2 \pi f k)$

Finally the estimated ARMA power spectrum is $[5,6]$

$$
\hat{P}_{A R M A}(f)=\frac{\hat{P}_{M A}(f)}{\left|1+\sum_{k=1}^{p} \hat{a}(k) e^{-j 2 \pi f k}\right|^{2}}
$$

\subsubsection{Selection Of ARMA Model Order}

One of the most important aspects of the use of ARMA method is the selection of the order $p$ and $q$. Much work has been done by various researchers on this problem and many experimental results have been given in the literature such as the papers presented by Akaike (1969, 1974), Chow (1972), Parzen (1974), Ulrych and Bishop (1975), Tong (1975, 1977), Jones (1976), Nuttall (1976), Berryman (1978), Bruzzone and Kaveh (1980), Kashyap (1980), and Rissanen (1983) [5,6]. One of the better known criteria for selecting the model order have been proposed by Akaike (1974), called the Akaike information criterion (AIC), is based on selecting the order that minimizes

$\operatorname{AIC}(p, q)=\ln \hat{\sigma}_{w p q}^{2}+\frac{2(p+q)}{N}$

where $\hat{\sigma}_{w p q}^{2}$ is the estimated variance of the linear prediction error. Note that the term $\hat{\sigma}_{w p q}^{2}$ decreases and therefore $\ln \hat{\sigma}_{w p q}^{2}$ also decreases as the order of ARMA method is increased. However, $2(p+q) / N$ increases with an increase in $p$ and $q$. In this situation, a minimum value is obtained for some $p$ and $q$ [7]. In this study, model orders $p$ and $q$ are taken as 10 by using equation (13).

\section{RESULTS AND DISCUSSION}

Doppler shift signal can be processed to achieve either a flow velocity waveform or a Doppler power spectrum. Clinically useful information can be extracted from these types of output. In this study, sonograms were obtained from 105 subjects, 38 of them have artery stenosis and 25 of them have artery occlusion. In Figure 2, artery flow sonograms recorded from 33-year-old healthy subject are presented. Artery flow sonograms in Figure 2(a), (b) are obtained by using FFT, and least squares modified Yule-Walker ARMA methods, respectively. When these sonograms are inspected, it is seen that there are some differences in the case of flow spectra. Spectral resolution is very low in the FFT sonogram comparing with least squares modified Yule-Walker ARMA sonogram. In the FFT sonogram some spurious frequencies are seen comparing with least squares modified Yule-Walker ARMA sonogram. Furthermore, the envelope of the sonograms of FFT methods are not seen clearly in comparison with least squares modified Yule-Walker ARMA method. The least squares modified Yule-Walker ARMA method offers a good quality sonogram output in terms of frequency resolution. 
In Figure 3, artery flow sonograms recorded from 35-year-old unhealthy subject suffering from artery stenosis are given. Artery flow sonogram in (a) using FFT method and in (b) using least squares modified Yule-Walker ARMA method. In Figure 4, artery flow sonograms recorded from 36-year-old unhealthy subject suffering from artery occlusion are presented. Artery flow sonograms in Figure 4(a), (b) are obtained by using FFT, and least squares modified Yule-Walker ARMA methods, respectively. Turbulent flow is observed in arteries due to stenosis and occlusion which are symptoms of artery disease. As it is seen from Figures 3 and 4, in the case of turbulent flow systole and diastole are not clear. Some misleading frequencies are seen on the FFT sonograms. Owing to this situation the sonograms obtained by using the FFT method do not have high frequency resolution. Doppler sonograms obtained by least squares modified YuleWalker ARMA method are more clear and have higher spectral resolution compared with FFT method at those areas where the flow speed of blood is high, fluctuated or insufficient.

When sonograms are examined, it is seen that FFT method has large variance (Figures 2(a), 3(a) and 4(a)). FFT method is based on a finite record of data, the frequency resolution of this method equals to the spectral width of the window length $N$, which is approximately $1 / N$. The principal effect of windowing that occurs when processing with FFT is to smear or smooth the estimated spectrum. Owing to smearing FFT method cannot resolve details in the studied spectrum that are separated by less than $1 / N$ in cycles per sampling interval. For this reason, $1 / N$ is called the spectral resolution limit of the FFT method. Furthermore, this method suffers from spectral leakage effects, due to windowing that are inherent in finite-length data records. Often, the spectral leakage masks weak signals that are present in the data. The basic limitation of the FFT method is the inherent assumption that the autocorrelation estimate is zero outside the window. This assumption severely limits the frequency resolution and the quality of the power spectrum estimate that is achieved. From another viewpoint, the inherent assumption in the FFT method estimate is that the data are periodic with period $N$. Neither one of these assumptions is realistic.

Parametric (model-based) methods do not require such assumptions. A model for the signal generation can be constructed with a number of parameters that can be estimated from the observed data. From the model and the estimated parameters, PSD can be computed. The modeling approach eliminates the need for window functions and the assumption that the autocorrelation sequence is zero outside the window. As a consequence, parametric PSD estimation methods avoid the problem of leakage and provide better frequency resolution than do the FFT method. Since nearly all data are corrupted by some amount of observation noise, ARMA method is the most appropriate method. By combining poles and zeros, ARMA method provides a more efficient representation, from the viewpoint of the number of model parameters, of the spectrum of a random process. Spectra with both sharp peaks and deep nulls can be modeled by ARMA equation.

As a result, least squares modified Yule-Walker method is used for estimating ARMA parameters of Doppler signals obtained from arteries in order to observe blood flow velocity in artery of healthy and unhealthy subjects suffering from artery stenosis and occlusion. The least squares modified Yule-Walker ARMA method has sharper response than other ARMA estimates. In Doppler sonograms obtained by using FFT 
methods, systole and diastole are not clear as shown in Figures 2(a), 3(a) and 4(a). On the other hand, least squares modified Yule-Walker ARMA method gives better performance for spectral resolution than FFT methods as shown in Figures 2(b), 3(b) and 4(b).

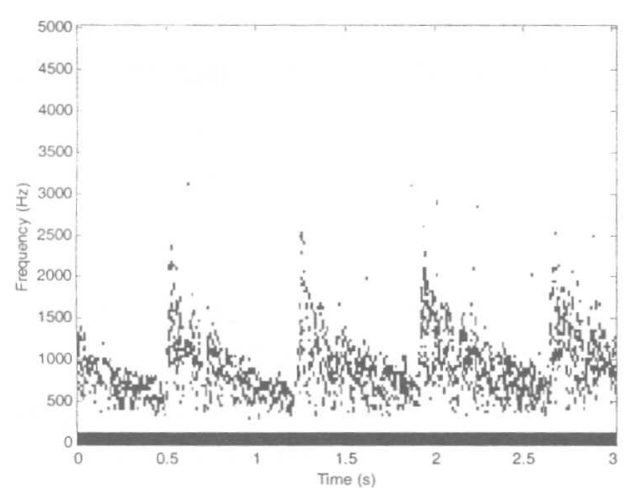

(a)

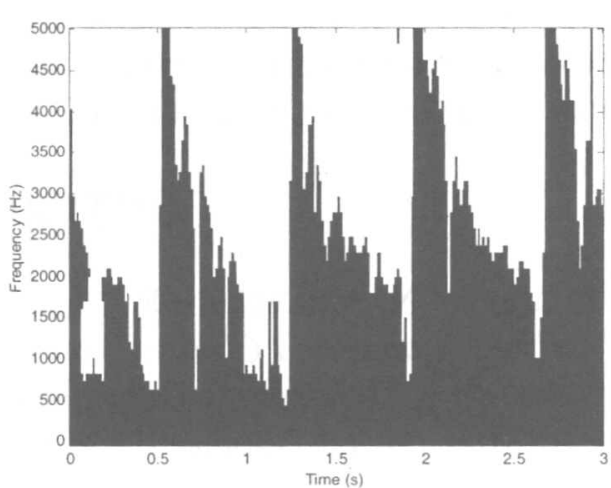

(b)

Figure 2. Doppler sonogram recorded from 33-year-old healthy subject: (a) FFT, (b) Least squares modified Yule-Walker ARMA.

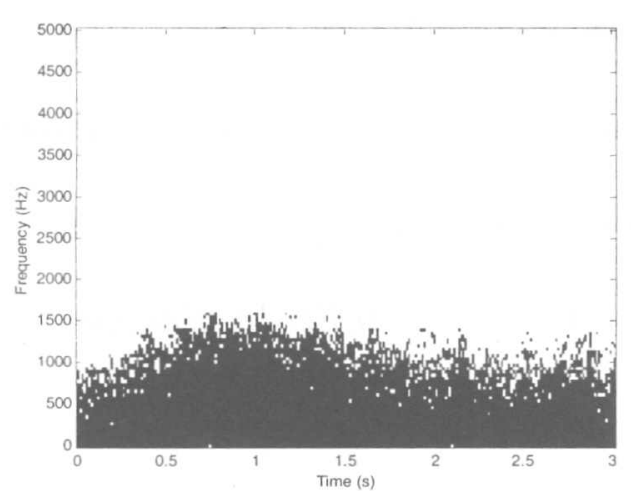

(a)

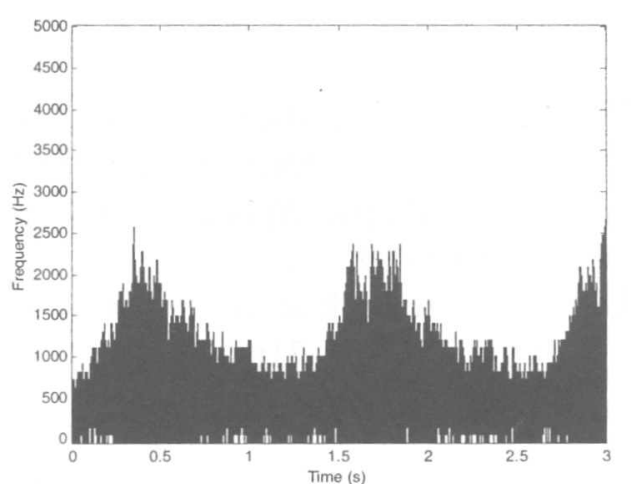

(b)

Figure 3. Doppler sonogram recorded from 35-year-old unhealthy subject (having artery stenosis): (a) FFT, (b)Least squares modified Yule-Walker ARMA.

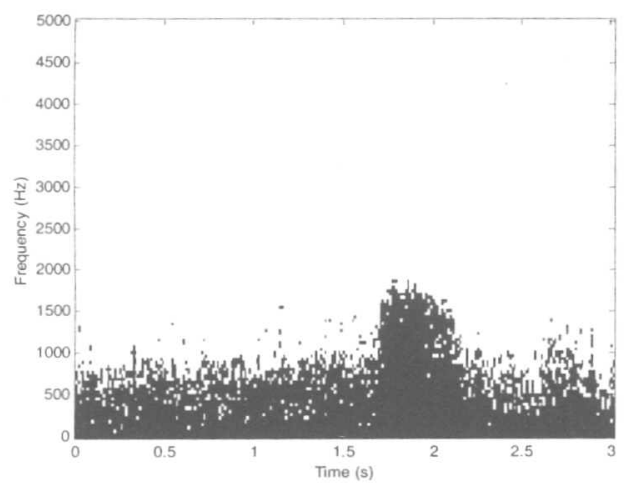

(a)

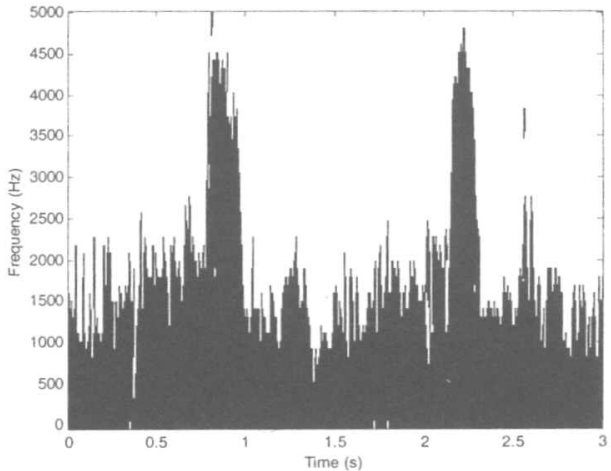

(b)

Figure 4. Doppler sonogram recorded from 36-year-old unhealthy subject (having artery occlusion): (a) FFT, (b)Least squares modified Yule-Walker ARMA. 


\section{CONCLUSION}

Doppler signals obtained from arteries were processed by using FFT, and least squares modified Yule-Walker ARMA methods. Interpretation and performance of these methods were compared in terms of their frequency resolution and the effects in clinical applications. Least squares modified Yule-Walker ARMA method's performance characteristics have been found extremely valuable for the use in clinical and research areas, because of their clear spectra.

\section{REFERENCES}

[1] D.H. Evans, W.N. McDicken, R. Skidmore, J.P. Woodcock, Doppler Ultrasound: Physics, Instrumentation and Clinical Applications, Wiley, Chicester, 1989.

[2] N.F. Güler, M.K. Kiymık, İ. Güler, Comparison of FFT and AR-based sonogram outputs of $20 \mathrm{MHz}$ pulsed Doppler data in real time, Comput. Biol. Med., 25, 383-391, 1995.

[3] N.F. Güler, M.K. Kıymık, İ. Güler, Autoregressive-based sonogram output of 20 MHz pulsed Doppler data, Medical Progress through Technology, 21, 105-110, 1995.

[4] İ. Güler, F. Hardalaç, S. Müldür, Determination of aorta failure with the application of FFT, AR and wavelet methods to Doppler technique, Comput. Biol. Med., 31, 229238, 2001.

[5] J.G. Proakis, D.G. Manolakis, Digital Signal Processing Principles, Algorithms, and Applications, Prentice Hall, New Jersey, 1996.

[6] S.M. Kay, S.L. Marple, Spectrum analysis - A modern perspective, Proceedings of the IEEE, 69, 1380-1419, 1981.

[7] H. Akaike, A new look at the statistical model identification, IEEE Transactions on Automatic Control, AC-19, 716-723, 1974. 